\title{
Electromagnetically Induced Transparency with Noisy Lasers
}

\section{Citation}

Xiao, Yanhong, Tun Wang, Maria Baryakhtar, Mackenzie Van Camp, Michael Crescimanno, Michael Hohensee, Liang Jiang, et al. 2009. Electromagnetically induced transparency with noisy lasers. Physical Review A 80(041805R).

\section{Published Version}

doi:10.1103/PhysRevA.80.041805

\section{Permanent link}

http://nrs.harvard.edu/urn-3:HUL.InstRepos:4310832

\section{Terms of Use}

This article was downloaded from Harvard University's DASH repository, and is made available under the terms and conditions applicable to Open Access Policy Articles, as set forth at http:// nrs.harvard.edu/urn-3:HUL.InstRepos:dash.current.terms-of-use\#OAP

\section{Share Your Story}

The Harvard community has made this article openly available.

Please share how this access benefits you. Submit a story.

Accessibility 


\title{
EIT with noisy lasers
}

\author{
Yanhong Xiao, ${ }^{1}$ Tun Wang, ${ }^{2,3}$ Maria Baryakhtar, ${ }^{4}$ Mackenzie Van Camp,${ }^{5}$ Michael Crescimanno, ${ }^{6}$ Michael \\ Hohensee, ${ }^{1,4}$ Liang Jiang, ${ }^{4}$ David F. Phillips, ${ }^{1}$ Mikhail D. Lukin, ${ }^{4}$ Susanne F. Yelin, ${ }^{2,7}$ and Ronald L. Walsworth ${ }^{1,4}$ \\ ${ }^{1}$ Harvard-Smithsonian Center for Astrophysics, Cambridge, MA, 02138 USA \\ ${ }^{2}$ Department of Physics, University of Connecticut, Storrs, CT 06269, USA \\ ${ }^{3}$ Institute for Theoretical Physics, University of Innsbruck, 6020 Innsbruck, Austria \\ ${ }^{4}$ Department of Physics, Harvard University, Cambridge, MA, 02138 USA \\ ${ }^{5}$ Department of Physics, Lawrence University, Appleton, WI 54912 USA \\ ${ }^{6}$ Department of Physics and Astronomy, Youngstown State University, Youngstown, OH 44555-2001 \\ ${ }^{7}$ ITAMP, Cambridge, MA, 02138 USA
}

(Dated: June 2, 2009)

\begin{abstract}
We demonstrate and characterize two coherent phenomena that can mitigate the effects of laser phase noise for Electromagnetically Induced Transparency (EIT): a laser-power-broadening-resistant resonance in the transmitted intensity cross-correlation between EIT optical fields; and a resonant suppression of the conversion of laser phase noise to intensity noise when one-photon noise dominates over two-photon-detuning noise. Our experimental observations are in good agreement with both an intuitive physical picture and numerical calculations. The results have wide-ranging applications to spectroscopy, atomic clocks and magnetometers.
\end{abstract}

PACS numbers: $42.50 . \mathrm{Gy}, 32.70 . \mathrm{Jz}, 42.50 . \mathrm{Md}$

When a laser interacts with a resonant medium, phase noise in the laser's optical field can be converted to intensity noise [1]. For Electromagnetically Induced Transparency (EIT) in atomic and other systems [2], such deleterious noise conversion is of great interest because of the wide application of EIT in atomic clocks [3, 4], magnetometers [5], quantum optics [6, 7] and quantum communications [8-10]. Laser phase-noise-to-intensitynoise conversion in the absence of atomic ground state coherence and its role in atomic-clock instability $[1,11-$ 13] and the effect of laser phase-noise on photon-photon correlations in atomic vapor cells $[6,7]$ have been studied previously. However, the role of EIT coherence in noise processes remains to be understood. In this Letter, we demonstrate and present intuitive models for two coherent noise phenomena, that can mitigate the conversion of laser phase noise to intensity noise in EIT media: (i) a laser-power-broadening-resistant resonance observed in intensity cross-correlations between the two EIT optical fields; and (ii) resonant suppression of phasenoise-to-intensity-noise conversion, which occurs when one-photon noise (driven by laser phase noise) dominates two-photon-detuning noise. We find good agreement between our experimental and theoretical investigations of these two coherent phenomena, which should enable improved EIT measurements with realistic (noisy) lasers in a wide variety of systems.

In our experiments, we used warm ${ }^{87} \mathrm{Rb}$ vapor for studies of both Zeeman EIT with degenerate ground states and hyperfine EIT with ground electronic states split by $\simeq 6.8 \mathrm{GHz}$. To observe EIT, we employed a Vertical Cavity Surface Emitting Laser (VCSEL) operating on the ${ }^{87} \mathrm{Rb} D_{1}$ line $(795 \mathrm{~nm})$ with a linewidth of $100 \mathrm{MHz}$, chosen for compatibility with real-world CPT atomic clocks [12] and magnetometers. The large phasenoise bandwidth allows us to neglect other noise sources such as laser intensity noise and polarization noise. For Zeeman EIT, we used the $5 S_{1 / 2}, F=2$ to $5 P_{1 / 2}, F^{\prime}=1$ transition; for hyperfine EIT, we used the $5 S_{1 / 2}, F=1,2$ to $5 P_{1 / 2}, F^{\prime}=2$ transition. The VCSEL was stabilized to a Dichroic Atomic Vapor Laser Lock [14]. We used vapor cells containing isotopically-enriched ${ }^{87} \mathrm{Rb}$ and heated to $40^{\circ} \mathrm{C}$, with a Ne buffer gas of either 2 Torr or 40 Torr. The optical path through the vapor cell was less than one absorption length, suppressing propagation effects and simplifying comparisons between measurements and models. The vapor cell was housed in high-permeability magnetic shielding to screen external magnetic fields; a homogeneous magnetic field was applied as necessary by an internal solenoid. For Zeeman EIT, a linearly polarized, monochromatic optical field was sent into the atomic medium. At the output, a quarter-wave plate and a polarizing beam splitter separated the circularly polarized components of the transmitted light. For each two-photon detuning, we recorded the AC transmission for $\sim 200 \mu$ s (longer times produced the same result) of the two polarizations and calculated $g^{2}(0)$ as defined below. For hyperfine EIT, we modulated the VCSEL at $3.42 \mathrm{GHz}$, half the ground state hyperfine splitting, using a low-noise $R F$ synthesizer; the \pm 1 sidebands formed the two EIT fields coupling the two hyperfine ground states. The polarization of the input optical fields was circularized using a quarter-wave plate, and the total transmitted power through the atomic medium was detected.

First, we discuss the power-broadening-resistant resonance observed in intensity cross-correlations of the two transmitted EIT optical fields, first observed in [6]. We find that the resonance lineshape has a dual structure containing power-broadened and power-independent components as shown in Fig. 1. The narrow central peak in the cross-correlation spectrum lies within a broader structure and has a linewidth approximately equal to 
the intrinsic decay rate of the ground state coherence, while the broader structure exhibits typical power broadening, as can be understood from an intuitive dark state model below. Here the cross-correlation is defined by $g^{(2)}(0) \equiv\left\langle\delta I_{1}(t) \delta I_{2}(t)\right\rangle / \sqrt{\left\langle\left(\delta I_{1}\right)^{2}\right\rangle\left\langle\left(\delta I_{2}\right)^{2}\right\rangle}$, where $\delta I_{1,2}$ are the intensity fluctuations of the two EIT fields at the output of the vapor cell. Positive (negative) $g^{(2)}(0)$ indicates correlation (anti-correlation) between fluctuations in the two transmitted field intensities.

A simple theoretical model captures the relevant physics of these intensity cross-correlation measurements. For an optically thin $\Lambda$-system with ground states $|a\rangle,|b\rangle$ and excited state $|e\rangle, g^{(2)}(0)$ is given by $[6]$ :

$$
g^{(2)}(0)=\frac{\left\langle\operatorname{Im}\left(\delta \rho_{e a}\right) \operatorname{Im}\left(\delta \rho_{e b}\right)\right\rangle}{\left.\sqrt{\left\langle\left(\operatorname{Im}\left(\delta \rho_{e a}\right)\right)^{2}\right\rangle\left\langle\left(\operatorname{Im}\left(\delta \rho_{e b}\right)\right)^{2}\right.}\right\rangle} .
$$

Here \langle\rangle represents an average over input field fluctuations; and $\operatorname{Im}\left(\delta \rho_{e a}\right)$ and $\operatorname{Im}\left(\delta \rho_{e b}\right)$ are the imaginary components of fluctuations away from steady state of the atomic density matrix elements corresponding to the optical coherences. In the dark-state basis, the numerator of Eq. (1), which determines the sign of $g^{(2)}(0)$, can be rewritten as

$$
G^{(2)}(0)=\left\langle\left(\operatorname{Im}\left(\delta \rho_{e+}\right)\right)^{2}\right\rangle-\left\langle\left(\operatorname{Im}\left(\delta \rho_{e-}\right)\right)^{2}\right\rangle,
$$

i.e., as the difference of the mean square of the optical coherence fluctuations involving the bright $(|+\rangle)$ and dark $(|-\rangle)$ states defined as $| \pm\rangle=\left(\Omega_{1,2}|a\rangle \pm \Omega_{2,1}|b\rangle\right) / \Omega$, where $\Omega_{1,2}$ are the complex Rabi frequencies for $|a, b\rangle \rightarrow|e\rangle$ transitions and $\Omega$ is the total two-photon Rabi frequency.

We modeled the dynamics in this simplified three-level $\Lambda$-system using a Hamiltonian expressed in the dark state basis:

$$
H=\Omega|e\rangle\langle+|+\Delta|+\rangle\langle-|-\delta| e\rangle\langle e|+\text { H. C. },
$$

where $\delta$ and $\Delta$ are the one- and two-photon detunings, and H. C. represents the Hermetian conjugate. We included phenomenological damping terms for atomic excited-state and intrinsic ground-state relaxation, with relaxation rates $\gamma$ and $\Gamma$, respectively. Three processes are present in this model: (i) the bright state is coupled to the excited state by the laser fields, incoherently optically pumping atoms into the dark state at a rate $\Omega^{2} / \gamma$; (ii) the dark state $|-\rangle$ and bright state $|+\rangle$ are coherently coupled at a rate given by the two photon detuning $\Delta$; and (iii) the dark and bright states are incoherently coupled at rate $\Gamma$.

The sign of $G^{(2)}(0)$ can be evaluated from the optical coherences in Eq. (2). The zeros of $G^{(2)}(0)$ then provide an estimate of the width of the cross-correlation resonance (see Fig. 1). Because there is no direct coherent coupling between $|e\rangle$ and $|-\rangle$, the $\rho_{e-}$ coherence can only be non-zero if coherence is established between the $|+\rangle$ and $|-\rangle$ states. Such ground state coherence $\left(\rho_{+-}\right)$is created from a population difference between the dark and bright states at a rate proportional to the two-photon
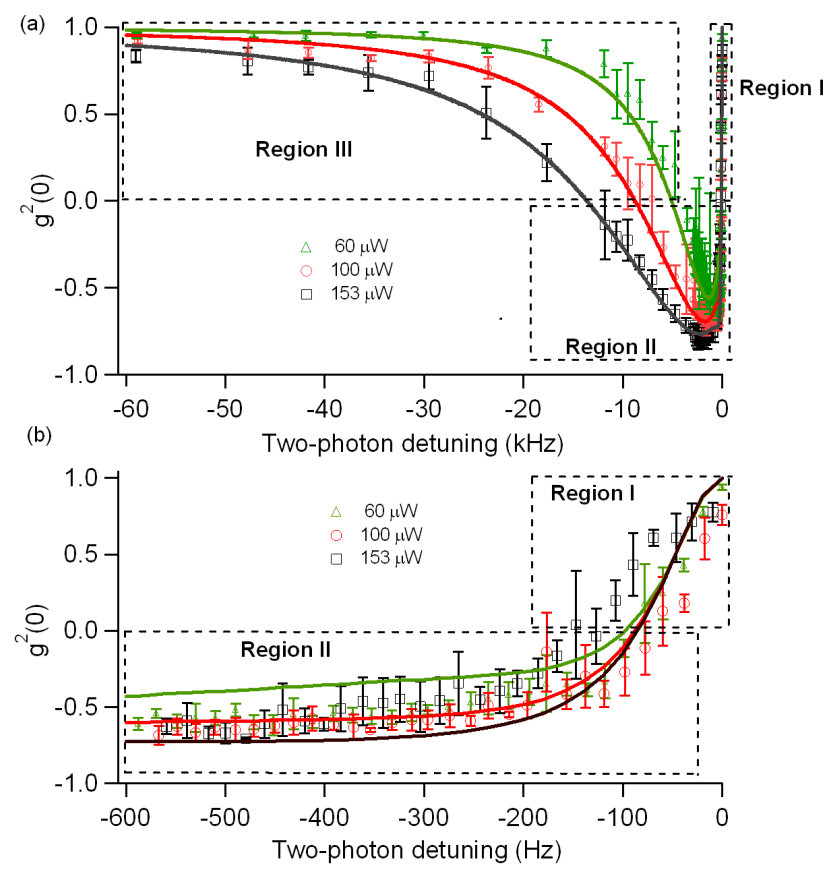

FIG. 1: Cross-correlation in the transmitted intensity fluctuations of Zeeman EIT vs. two-photon detuning as measured (symbols) and calculated (lines). In (a) regions I-III of correlation, anti-correlation and return to correlation described in the text are visible, while (b) shows the central region of (a) near zero two-photon detuning. The linewidth of the central peak is approximately the same for 60 and $100 \mu \mathrm{W}$ data, and broader for $153 \mu \mathrm{W}$ data which was taken at higher magnetic gradient, showing that intrinsic decoherence determines the narrow resonance width. Only negative detuning is shown, as the experimental results are symmetric with two-photon detuning. 2 Torr Ne buffer gas and $3 \mathrm{~mm}$ laser beam diameter. In the calculation, an intrinsic decay rate of $\approx 2 \pi \times 100 \mathrm{~Hz}$ was used for all laser powers.

detuning $\Delta$. When $|\Delta| \ll \Gamma \ll \Omega^{2} / \gamma$, atoms remain almost entirely in the dark state as decoherence suppresses $\rho_{+-}$and hence $\rho_{e-}$, and from Eq. 2 leads to positive correlations between the intensity of the two transmitted EIT optical fields (see region I of Fig. 1). When $\Gamma<|\Delta| \ll \Omega^{2} / \gamma$, dark state population dominates over bright state population and it drives the $\rho_{+-}$coherence faster than it decoheres. This allows $\rho_{e-}$ to dominate $\rho_{e+}$, leading to anti-correlations in the transmitted field intensities (region II of Fig. 1). The cross-over between region I and II sets the width of the narrow central peak and occurs for $\Delta \approx \Gamma$, which is independent of laser power. For $|\Delta|>\Omega^{2} / \gamma \gg \Gamma$, the atomic population oscillates between the bright and dark states faster than optical pumping repopulates the dark state, which reduces the rate at which $\rho_{+-}$is produced, and hence returns the system to a positive $G^{(2)}(0)$ (region III).

Fig. 1 shows example cross-correlation $\left(g^{(2)}(0)\right)$ data for Zeeman EIT. Regions I through III described above are clearly seen, with the transition from region I to II 
(a)

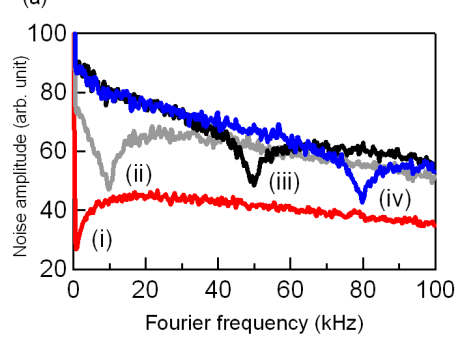

(b)

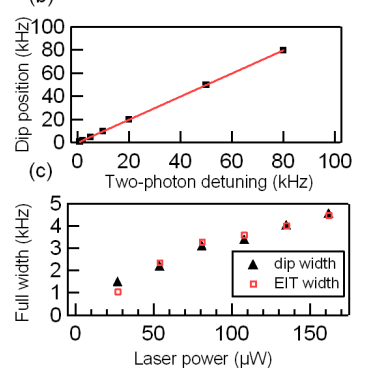

FIG. 2: (a) Measured noise spectra of total transmitted power for hyperfine EIT. Two-photon detunings of (i) $1 \mathrm{kHz}$, (ii) 10 $\mathrm{kHz}$, (iii) $50 \mathrm{kHz}$, and (iv) $80 \mathrm{kHz}$. (b) Dip center frequencies vs. two-photon detuning (symbols); line represents dip center = detuning. (c) Widths of dips and EIT resonances are equal at all input laser powers. (Two-photon detuning $=50 \mathrm{kHz}$.) Laser power $\approx 170 \mu \mathrm{W}$ for plots (a) and (b). $0.85 \mathrm{~mm}$ beam size and 2 Torr Ne buffer gas for (a-c).

determining the width of the power-broadening-resistant resonance. A low buffer gas pressure $\mathrm{Rb}$ vapor cell (2 Torr $\mathrm{Ne}$ ) was used in the measurements shown in Fig. 1 to reduce pressure broadening and hence effects from the second excited state [15]. The measured half width at half maximum of the central peak of the cross-correlation resonance is $\approx 100 \mathrm{~Hz}$ for all laser power (Fig. 1b), which is consistent with the extrapolated zero-power EIT linewidth for this system, and is limited by residual magnetic field inhomogeneity as shown by the slight broadening of the $153 \mu \mathrm{W}$ data under larger field gradient than the other two laser powers; whereas the conventional, power-broadened EIT linewidth is much greater. These measurements demonstrate that the central crosscorrelation peak is immune to power-broadening. Note that for lower laser power, insufficient to optically pump atoms into the dark state, complete anti-correlation between the transmitted field intensities is not reached, which is consistent with the picture presented above. An analytical model using above analysis and phenomenologically including the coherent return of atoms [16] to the laser beam reproduces the observed cross-correlation results (lines in Fig. 1), and will be presented elsewhere.

Potential applications of the power-broadeningresistant $g^{(2)}(0)$ resonance include measure of the intrinsic decoherence rate even with high laser power, and atomic clocks and magnetometers for which there is often a trade-off between a narrow resonance and sufficient signal-to-noise-ratio (SNR) because of power broadening. While the data reported above relies upon Zeeman EIT, the analysis is for a general three-level system and therefore is applicable to hyperfine EIT and atomic clocks. Using data shown in Fig. 1, and generalizing to orthogonal linear polarization hyperfine EIT [17], we estimate the stability to be $3 \times 10^{-11} \tau^{-1 / 2}$, comparable to current CPT clock performance [18]. This stability can be further improved for the cross-correlation resonance with in- creased laser power which allows for higher SNR without broadening the resonance, potentially enabling a superior atomic reference.

Next, we discuss the second topic of this paper: the resonant suppression of laser phase-noise-to-intensity-noise conversion in EIT media, governed by the relative importance of one-photon and two-photon-detuning noise. Figure 2(a) shows examples of measured intensity noise spectra for hyperfine EIT at several two-photon detunings (2 Torr Ne buffer gas, $170 \mu \mathrm{W}$ laser power). In each case, the intensity noise drops at a Fourier frequency equal to the two-photon detuning (Fig. 2[b]), with a linewidth equal to the EIT linewidth (Fig. 2[c]) and a lineshape determined by diffusion of atomic coherence in and out of the laser beam [16]. Numerical analysis indicates that the reduced intensity noise near two-photon resonance results from phase correlations between the two EIT optical fields [19]. When the fields' two-photon detuning, $\Delta$, is nonzero but much smaller than the excited state linewidth or the laser linewidth, Fourier components of phase fluctuations in one optical field that are offset by $\Delta$ from that field's central frequency will form EIT with the other optical field; this EIT will suppress absorption and conversion of laser phase noise to intensity noise within the EIT linewidth.

This observed resonant drop in laser phase-noise-tointensity-noise conversion for an EIT system is in striking contrast to the enhanced intensity noise observed before $[4,20]$. The qualitatively different behavior in the two cases can be understood by noting that the effect of laser phase noise in a two-level system is analogous to two-photon-detuning noise in EIT. We find experimentally that either a resonant peak or dip can appear in EIT intensity noise spectra, determined by the relative importance of one-photon noise (arising from laser phase noise) and two-photon-detuning noise (due to phase noise in the RF signal used to modulate the laser for hyperfine EIT, or magnetic field noise for Zeeman EIT [21]). In particular, when an EIT system is subjected to sufficient two-photon-detuning noise, the EIT phase correlations arising from laser phase noise are destroyed, and the resonant dip in the intensity noise spectrum is transformed into a noise peak.

We performed two experiments to illustrate the importance for EIT noise spectra of the relative magnitude of one-photon noise and two-photon-detuning noise. First we measured hyperfine EIT noise spectra in a 2 Torr Ne buffer gas cell, which had significant one-photon noise induced by the VCSEL's phase noise, but small two-photon-detuning noise $(-95 \mathrm{dBc} / \mathrm{Hz}$ at $10 \mathrm{kHz}$ offset from the $3.42 \mathrm{GHz}$ signal modulating the VCSEL). With this set-up, we found a dip in the EIT intensity noise spectrum (Fig. 3[a], curve [i]). With sufficient added phase noise to bring the two-photon-detuning noise to dominance $(-75 \mathrm{dBc} / \mathrm{Hz}$ at $10 \mathrm{kHz}$ offset), conventional EIT for the transmitted fields was unaffected, but the noise dip was transformed into a peak (Fig. 3[a], curve [ii]). Next we employed a high pressure buffer gas cell 
(a)
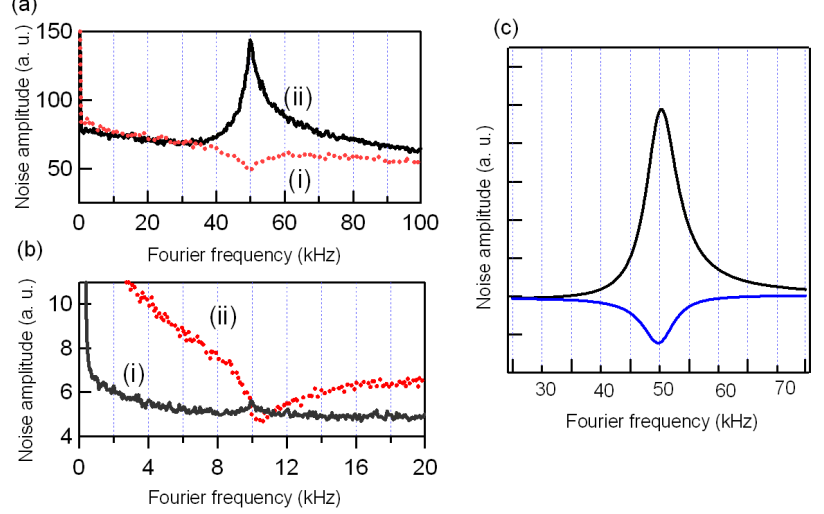

FIG. 3: (a-b) Measured transition between resonant suppression (i) and enhancement (ii) of laser phase-noise-to-intensitynoise conversion in hyperfine EIT. (See text for experimental details.) (c) Examples of calculated noise spectra for no two-photon phase noise (dip) and extra phase noise (peak) at $\Delta=50 \mathrm{kHz}$.

(40 Torr Ne), which broadened the one-photon resonance linewidth and hence reduced the conversion of laser phase noise to one-photon intensity noise [22], such that twophoton-detuning noise dominated without adding extra noise to the RF modulation signal. With this set-up, we observed a noise peak for hyperfine EIT on one-photon resonance (Fig. 3[b], curve [i]). A noise dip re-emerged by detuning the laser from one-photon resonance by less than half a linewidth, which increased the conversion of laser phase noise to one-photon intensity noise, so that it dominated the effect of two-photon-detuning noise (Fig. 3[b], curve [ii]). Our numerical modeling agrees with the observed EIT noise spectra described above (Fig. $3[\mathrm{c}]$ ): noise spectra peaks and dips are found at a Fourier frequency equal to the two-photon detuning; dips always appear in the absence of two-photon detuning noise, while peaks appear when sufficient two-photon detuning noise is added. Model lineshapes are Lorentzian instead of exhibiting the sharper structure of experimental data due to lack of coherence return in the simple model. A small offset of the observed peak and dip lo- cation may be attributed to the sloped underlying intensity noise background determined by phase noise spectra of the laser and the synthesizer and their frequencydependent conversion into laser intensity noise, which necessitates further investigation.

These results show that transmitted intensity noise spectra can be used as a broadband measure of EIT resonance location without scanning the two-photon detuning. The location and linewidth of the noise dip or peak indicates the EIT resonance frequency and linewidth directly. In addition, the noise peak or dip is detectable for two-photon detunings much greater than the narrow EIT linewidth. Combining these noise spectra techniques with a broadband laser source could provide extremely broadband coverage [23]. Off-resonant detection of the two-photon EIT resonance via noise spectra could also be used in sensing or imaging applications where other resonant effects (e.g., absorption) are of concern. Additionally, we note the possibility that input laser phase noise could serve as a seed field to amplify quantum fluctuations in an EIT medium and thereby enable improved noise spectroscopy [24]. Finally, this technique has broad applicability due to the simple nature of its coupling of laser noise to the evolution of ground state coherence. As such this technique should be applicable to many coherent media including EIT in rare-earth doped crystals and color centers in diamond.

In conclusion, we demonstrated and characterized two coherent phenomena associated with EIT and laser phase noise: a laser-power-broadening-resistant resonance in the transmitted intensity cross-correlation of EIT optical fields; and resonant suppression or enhancement of laser phase-noise-to-intensity-noise conversion, governed by the relative magnitude of one-photon noise and twophoton-detuning noise. The present results may enable improved EIT measurements with realistic (noisy) lasers, and hence be useful for applications such as atomic clocks, magnetometers, quantum optics and imaging.

We are grateful to I. Novikova, J. Kitching and J. Vanier for useful discussions. This work was supported by ONR, the Smithsonian Institution and NSF.
[1] J. C. Camparo, et al., Phys. Rev. A 59, 728 (1999).

[2] M. Fleischhauer, et al., Rev. Mod. Phys. 77, 633 (2005).

[3] J. Vanier, Appl. Phys. B 81, 421 (2005).

[4] A. Godone, et al., Phys. Rev. A 66, 063807 (2002).

[5] M. Martinelli, et al., Phys. Rev. A 69, 043809 (2004).

[6] V. A. Sautenkov, et al., Phys. Rev. A 72, 065801 (2005); G. O. Ariunbold, et al., quant-ph/0603025 (2006).

[7] L. S. Cruz, et al., Europ. Phys. J. D 41, 531 (2007); C. L. G. Alzar, et al., Europhys. Lett. 4, 485(2003).

[8] M. D. Lukin, Rev. Mod. Phys. 75, 457 (2003).

[9] A. Sinatra, Phys. Rev. Lett. 97, 253601 (2006).

[10] M. T. L. Hsu, et al., Phys. Rev. Lett. 97, 183601 (2006).

[11] T. Yabuzaki, et al., Phys. Rev. Lett. 67, 2453 (1998).
[12] J. Kitching, et al., J. Opt. Soc. B 18, 1676 (2001).

[13] J. Kitching, et al., Opt. Lett. 26, 1507 (2001).

[14] K. L. Corwin, et al., Appl. Opt. 37, 3295 (1998).

[15] I. Novikova, et al., J. Opt. Soc. Am. B 22, 44 (2005).

[16] Y. Xiao, et al., Phys. Rev. Lett. 96, 043201(2006).

[17] T. Zanon et al., Phys. Rev. Lett. 94, 193002 (2005).

[18] J. Vanier, Appl. Phys. B 81, 421 (2005).

[19] S. E. Harris, Phys. Rev. Lett. 70, 552 (1993); M. Fleischhauer, et al., Phys. Rev. A 51, 2430 (1995); A. F. Huss, et al., Phys. Rev. Lett. 93, 223601 (2004); E. E. Mikhailov, et al., Phys. Rev. A 74, 013807 (2005); D. S. $\mathrm{Yu}$, et al., Phys. Rev. Lett. 98, 050801 (2007).

[20] J. C. Camparo, et al., Phys. Rev. A. 58, 3873 (1998). 
[21] The phase noise between two independent lasers exciting EIT creates both one- and two-photon detuning noise.

[22] J. G. Coffer, et al., Phys. Rev. A 65, 033807 (2002).

[23] X. Xu, et al., Nature Physics 4, 125 (2008).
[24] S. A. Crooker, et al., Nature 431, 49 (2004); N. Muller and A. Jerschow, Proc. Nat. Acad. Sci. 103, 6790 (2006). 